> Les anticorps monoclonaux utilisés en oncologie exercent des effets antitumoraux directs qui aboutissent à la mort des cellules tumorales. Ceux-ci mettent en jeu un grand nombre de mécanismes, allant de l'induction d'une apoptose au recrutement de cellules effectrices de l'immunité innée. Cependant, ces anticorps sont également capables d'induire des effets à long terme contre les tumeurs. Ceux-ci sont fondés sur l'induction d'une immunité adaptative antitumorale où les lymphocytes $\mathrm{TCD}^{+}$et $\mathrm{CD} 8^{+}$jouent un rôle central. Divers modèles expérimentaux précliniques, renforcés par quelques observations cliniques, ont démontré ainsi que, loin d'être les acteurs d'une seule immunothérapie passive, les anticorps monoclonaux utilisés en oncologie sont de véritables molécules à effet vaccinal, et induisent une mémoire immunitaire qui pourrait être à l'origine des réponses cliniques à long terme parfois observées. Cette possibilité de renforcer l'immunosurveillance des tumeurs peut également bénéficier de l'utilisation, éventuellement conjointe, d'anticorps antagonistes bloquant des molécules comme CTLA-4 (cytotoxic T-lymphocyte antigen 4) ou PD-1 (programmed cell death protein-1) qui jouent un rôle central dans l'inhibition des réponses immunes antitumorales. Enfin, ce nouveau concept d'anticorps inducteurs de réponses adaptatives antitumorales de type mémoire nous oblige à revoir l'articulation temporelle entre traitement par anticorps, chimiothérapie, radiothérapie et utilisation d'immunomodulateurs de la réponse immune. <

\section{Le double visage des anticorps monoclonaux en oncologie}

Immunité passive et vaccination

Claire Deligne, Jean-Luc Teillaud

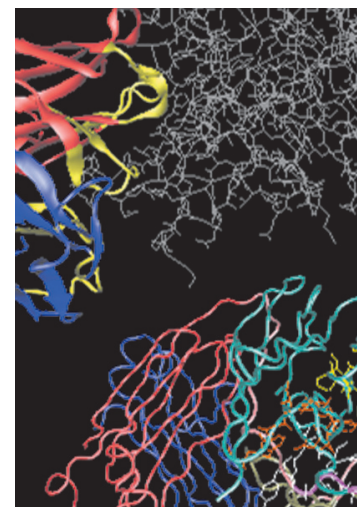

Centre de recherche des Cordeliers, Inserm U872, université Paris-Descartes, université Pierre et Marie Curie, 15 , rue de l'École de médecine, 75270 Paris Cedex 06, France. claire.deligne@gmail.com jean-luc.teillaud@crc.jussieu.fr

Les anticorps monoclonaux (Acm) ont été utilisés pour le traitement des cancers dès le début des années 1980, avec le travail pionnier de l'équipe de Ronald Lévy (université de Stanford, États-Unis). Cette équipe a traité en 1982 un patient présentant un lymphome avec un $\mathrm{Acm}$ dirigé contre l'immunoglobuline présente à la surface des cellules de ce lymphome (Acm anti-idiotype) [1]. Seize Acm ont reçu une autorisation de mise sur le marché (AMM) en Europe et/ou aux États-Unis pour le traitement des cancers (deux d'entre eux, l'édrécolomab et le gemtuzumab ont été cependant retirés en raison de leur manque d'efficacité et d'un rapport bénéfice/risque défavorable) $($ Tableau I)1 $\rightarrow$ (17]. D’autres anticorps

$(\rightarrow)$ Voir $\mathrm{m} / \mathrm{s} \mathrm{n}^{\circ} 12$ vol. 25, décembre 2009 ont une indication en oncologie en République populaire de Chine, en Inde et dans d'autres pays du Sud-Est asiatique, ainsi qu'en Amérique latine (par exemple, Cuba) et en Amérique du Sud (par exemple, Brésil) (Tableau 1). L'effet antitumoral de ces Acm repose sur des mécanismes parfois très différents, dépendants des molécules ciblées : une première catégorie d'Acm est dirigée contre les cellules tumorales, une seconde contre des molécules modulant l'environnement tissulaire et cellulaire des tumeurs (Figure 1). La vision initiale qui prévalait lorsque ces anticorps ont été développés était celle d'une sorte de «sérothérapie monoclonale », une immunothérapie passive [18]. Celle-ci reposait sur les capacités de ces anticorps à bloquer l'activation et/ou la prolifération des cellules tumorales (en ciblant par exemple des récepteurs de facteurs de croissance comme le récepteur de l'EGF [epidermal growth

${ }^{1}$ Voir le numéro thématique consacré par médecine/sciences en décembre 2009 aux « anticorps monoclonaux en thérapeutique ». 


\begin{tabular}{|c|c|c|c|c|}
\hline Nom (nom de marque) & $\begin{array}{l}\text { Espèce/format } \\
\text { (isotype) }\end{array}$ & Date AMM* & Cible & Indication ${ }^{\circ}$ \\
\hline Edrécolomab (Panorex ${ }^{\circledR}$ ) & Souris $(\operatorname{IgG} 2 a, \kappa)$ & $1995^{*}(\text { All. })^{\circ 0}$ & EpCAM & $\mathrm{CRC}^{* *}$ \\
\hline Rituximab (Mabthera $\left.{ }^{\circledR}\right)^{+}$ & Chimérique $(\operatorname{IgGl}, \kappa)$ & 1997 & $\mathrm{CD} 20$ & $\mathrm{LNH}^{\star *}$ \\
\hline Trastuzumab (Herceptine ${ }^{\circledR}$ ) & Humanisé $(\operatorname{IgGl}, \kappa)$ & 1998 & HER2/neu & Cancer du sein \\
\hline Gemtuzumab (Mylotarg ${ }^{\circledR}$ ) & Humanisé $(\lg G 4, \kappa)^{\#}$ & $2000^{*}$ & CD33 & $\operatorname{LAM}^{\star \star}$ \\
\hline $\begin{array}{l}\text { Alemtuzumab } \\
\left(\text { MabCampath }{ }^{\circledR}\right)\end{array}$ & Humanisé $(\lg G l, \kappa)$ & 2001 & CD52 & $\mathrm{LLC}^{\star \star}$ \\
\hline $\begin{array}{l}\text { Ibritumomab tiuxetan } \\
\left(\text { Zevalin }^{\circledR}\right)\end{array}$ & $\begin{array}{c}\text { Souris }(\operatorname{IgGl}, \kappa) \\
\text { (couplé à : Ind } 111 / \mathrm{Y}^{99} \text { ) }\end{array}$ & 2002 & CD20 & $\mathrm{LNH}^{\star \star}$ \\
\hline $\begin{array}{l}\text { Tositumomab-1 }{ }^{131} \\
\left(\text { Bexxar }^{\circledR}\right)\end{array}$ & $\begin{array}{l}\text { Souris }(\operatorname{IgG} 2 \mathrm{a}, \kappa) \\
\text { (couplé à : }\left.\right|^{131} \text { ) }\end{array}$ & 2003 & CD20 & $\mathrm{LNH}^{\star \star}$ \\
\hline $1^{131}$-Vivatuxin ${ }^{\circledR} / /^{131}$ - $\operatorname{chTNT}^{\circledR}$ & Chimérique IgG & $2003(\mathrm{RPC})^{\circ}$ & NT $\mathrm{Ag}^{\star \star \star}$ & $\begin{array}{l}\text { Cancer du } \\
\text { poumon }\end{array}$ \\
\hline Cétuximab (Erbitux ${ }^{\circledR}$ ) & Chimérique (IgGl, א) & 2004 & $\varepsilon G F-R$ & $\begin{array}{l}\text { Cancer tête } \\
\text { et cou }\end{array}$ \\
\hline Bévacizumab (Avastin ${ }^{\circledR}$ ) & Humanisé $(\operatorname{IgGl}, \kappa)$ & 2004 & VEGF-A & $\mathrm{CRC}^{\star \star}$ \\
\hline $\begin{array}{l}\text { Métuximab } \\
\left(\left.\right|^{131} \text {-Licartin }{ }^{\circledR}\right)\end{array}$ & Chimérique $\left(\text { Fab }^{\prime}\right)_{2}$ & 2005 (RPC) & CD147 & $H P C^{\star *}$ \\
\hline $\begin{array}{l}\text { Nimotuzumab }\left(\text { TheraCIM }^{\circledR}\right) \\
\left(\text { BIOMab EGFR }^{\circledR}\right) \\
\left(\text { CIMAHer }^{\circledR}\right)\end{array}$ & Humanisé $(\lg G l, \kappa)$ & $\begin{array}{c}2005^{++} \\
\text {(Singapour) } \\
\text { (Inde) } \\
\text { (Cuba) }\end{array}$ & $\varepsilon G F-R$ & $\begin{array}{c}\text { Cancer tête } \\
\text { et cou, } \\
\text { nasopharyngé, } \\
\text { gliome }\end{array}$ \\
\hline Panitimumab $\left(\right.$ Vectibix $\left.^{\circledR}\right)$ & Humain $(\operatorname{IgG} 2, \kappa)$ & 2006 & $\varepsilon G F-R$ & CRC \\
\hline Catumaxomab (Removab ${ }^{\circledR}$ ) & $\begin{array}{l}\text { Souris } \lg G 2 a / \\
\text { rat } \lg G 2 b^{\circ 00}\end{array}$ & 2009 & $\begin{array}{c}\text { EpCAM/ } \\
\text { CD3 }\end{array}$ & $\begin{array}{l}\text { Ascite } \\
\text { maligne }\end{array}$ \\
\hline Ofatumumab $\left(\right.$ Arzerra $\left.^{\circledR}\right)$ & Humain $(\operatorname{IgGl}, \kappa)$ & 2009 & $\mathrm{CD} 20$ & LLC \\
\hline Dénosumab (Prolia $\left.{ }^{\circledR}\right)$ & Humain $(\operatorname{Ig} G 2, \kappa)$ & 2010 & RANK-L & $\begin{array}{l}\text { Métastases } \\
\text { osseuses }\end{array}$ \\
\hline Ipilimumab $\left(\right.$ Yervoy $\left.^{\circledR}\right)$ & Humain $(\operatorname{IgGl}, \kappa)$ & 2011 & CTLA-4 & Mélanome \\
\hline $\begin{array}{l}\text { Brentuximab vedotin } \\
\left(\text { Adcentris }^{\circledR}\right)\end{array}$ & $\begin{array}{l}\text { Chimérique } \\
(\lg G l, \kappa)^{\# \#}\end{array}$ & 2011 & CD30 & $\begin{array}{l}\mathrm{LH} \\
\mathrm{ALCL}^{* *}\end{array}$ \\
\hline Pertuzumab (Perjeta ${ }^{\circledR}$ ) & Humanisé $(\lg G l, \kappa)$ & 2012 & HER2/neu & $\begin{array}{l}\text { Cancer du } \\
\text { sein }\end{array}$ \\
\hline
\end{tabular}

Tableau I. Anticorps monoclonaux à usage thérapeutique (oncologie) (voir aussi [17]). ${ }^{*}$ Année du $1^{\text {er }}$ enregistrement EMA (European medicines agency, ex-EMEA, Union Européenne) ; FDA (Food and drug administration, États-Unis) ; SFDA (State food and drug administration, République populaire de Chine).

'Seule l'indication initiale est présentée (à l'exception du dénosumab dont la l lre indication a été l'ostéoporose post-ménopause).

${ }^{+}$Rituxan ${ }^{T M}$ aux États-Unis.

${ }^{\star *} \mathrm{ALCL}$ : lymphome anaplasique à grandes cellules; CRC : cancer colorectal ; HPC : hépatocarcinome ; LAM : leucémie aiguë myéloïde ; LH : lymphome hodgkinien; LLC : leucémie lymphoïde chronique ; LNH : lymphome non hodgkinien.

\# Immunoconjugué couplé à l'ozogamycine.

* Retiré du marché (édrecolomab : retiré du marché après deux essais de phase III publiés ne montrant aucun bénéfice ; gemtuzumab retiré du marché en 2010 en raison d'un rapport bénéfice/risque défavorable).

${ }^{\circ}$ All. : République fédérale d’Allemagne ; RPC : République populaire de Chine.

${ }^{\star \star \star}$ NT Ag : necrotic tumor antigen ; TNT : tumor necrotic therapy.

++ Statut de médicament orphelin accordé par la FDA et l'EMA pour le traitement des gliomes et par l'EMA pour le traitement de cancers du pancréas.

${ }^{\circ}$ Quadrome : anticorps bispécifique issu de la fusion de deux hybridomes.

\# Immunoconjugué couplé au mono-méthyle d'auristatine $\varepsilon$ (MMAE). 

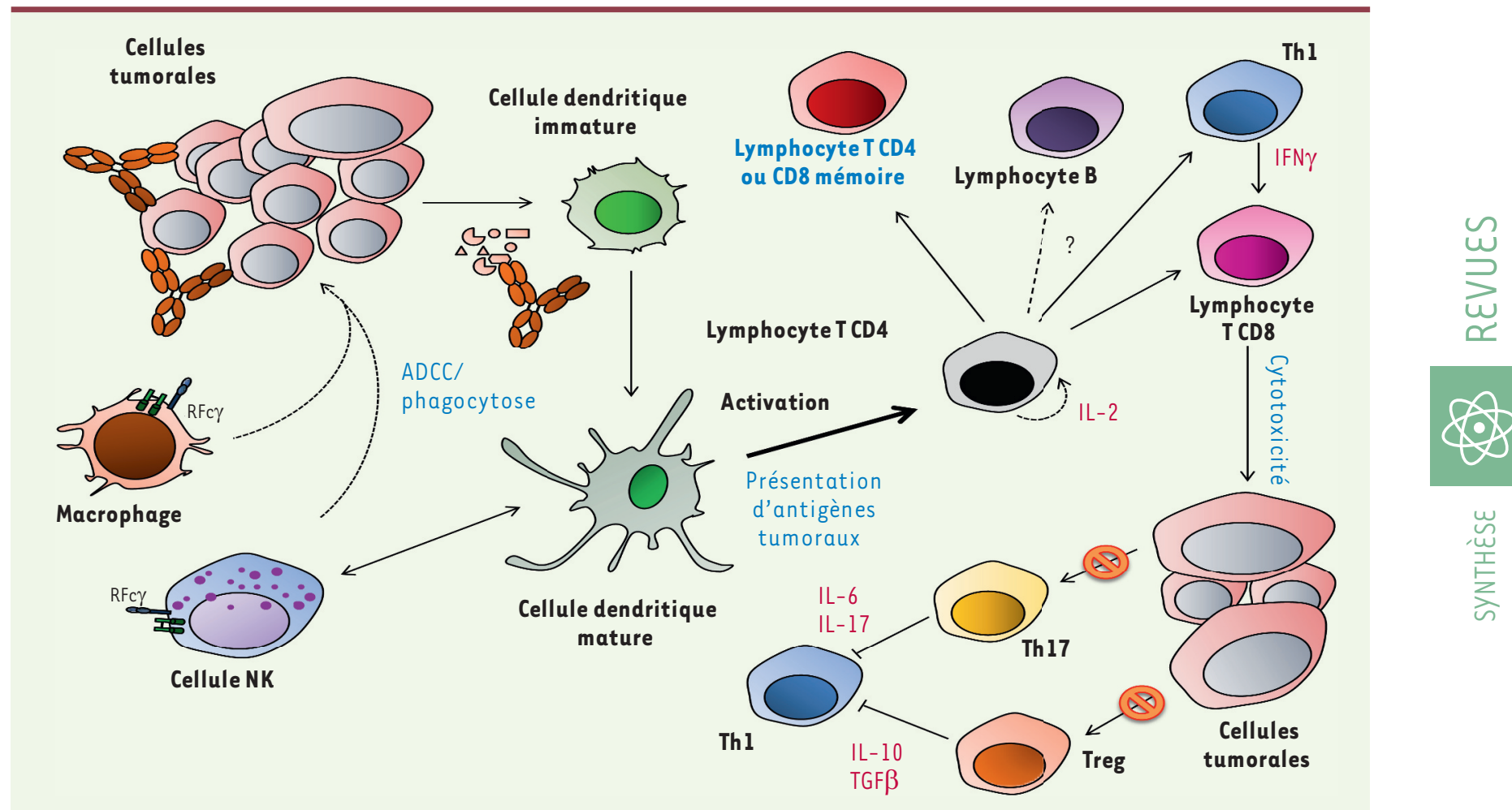

Figure 1. Mécanismes d'action des anticorps monoclonaux à usage thérapeutique en oncologie. Le blocage de molécules d'adhérence comme EpCAM, impliquées dans la migration des cellules tumorales et les métastases, n'est pas figuré. Le blocage du VEGF, impliqué dans la formation des vaisseaux tumoraux, n'est également pas indiqué. ADCC : cytotoxicité cellulaire dépendante des anticorps ; CDC : cytotoxicité dépendante du complément ; RFc $\gamma$ : récepteurs pour la région Fc des IgG ; Th : lymphocytes T auxiliaires.

factor] ou HER2/neu [erbB-2, human EGFR2, epidermal growth factor receptor 2]), à induire une apoptose, même discrète (anti-CD20), ou à interférer avec les capacités d'adhérence des cellules tumorales (Acm ciblant Ep (AM, epithelial cell adhesion molecule). Cette immunothérapie passive reposait également sur l'activation des mécanismes effecteurs de l'immunité, comme la cytotoxicité cellulaire dépendante d'anticorps (ADCC, antibody-dependent cell cytotoxicity), la phagocytose, ou l'activation de la voie classique du complément aboutissant à la formation d'un complexe d'attaque membranaire responsable de la mort des cellules tumorales (Figure 1). De nombreux efforts d'ingénierie ont été entrepris au cours de la dernière décennie pour optimiser ces fonctions effectrices, principalement en manipulant la région constante $(F c)$ de ces anticorps (qui sont principalement des Fc d'lgGl humaines) [19], en couplant les anticorps à des drogues (par exemple au mono-méthyle d'auristatine $\varepsilon$, MMAE) ou en les rendant bispécifiques, ce qui permet le recrutement de cellules de l'immunité comme les lymphocytes T (par exemple, le blinatumomab, un anticorps anti-CD3 et anti-CD19).

\section{Les anticorps monoclonaux en oncologie : de la destruction des cellules tumorales à la mobilisation de l'immunité adaptative}

Cependant, au cours de ces dernières années, le concept d'une surveillance des tumeurs par le système immunitaire s'est enrichi grâce à des études de grandes cohortes de patients atteints de divers cancers parmi les plus fréquents (colorectaux et pulmonaires) [2]. Outre leurs effets antitumoraux directs et rapides, les Acm pourraient avoir un impact important sur l'immunosurveillance des tumeurs en permettant à l'immunité adaptative de se développer pour exercer un contrôle accru des tumeurs; ceci rendrait compte des réponses observées à long terme chez certains patients traités par des Acm (Figure 2). L'utilisation du trastuzumab (anti-HER2/neu) chez des patientes atteintes d'un cancer du sein métastatique est généralement associée à une présence accrue de lymphocytes T CD4 ${ }^{+}$spécifiques de peptides dérivés de HER2/neu [3]. De fait, l'effet thérapeutique du trastuzumab dépend de sa capacité à interférer avec la signalisation intracellulaire due à $H \varepsilon R 2 / n e u$, mais repose aussi très probablement sur sa capacité à induire des réponses immunitaires innée et adaptative antitumorales.

Les Acm induisent une réponse adaptative antitumorale spécifique : les arguments expérimentaux

Différentes expériences réalisées chez des souris porteuses de tumeurs ont mis en lumière le rôle essentiel 


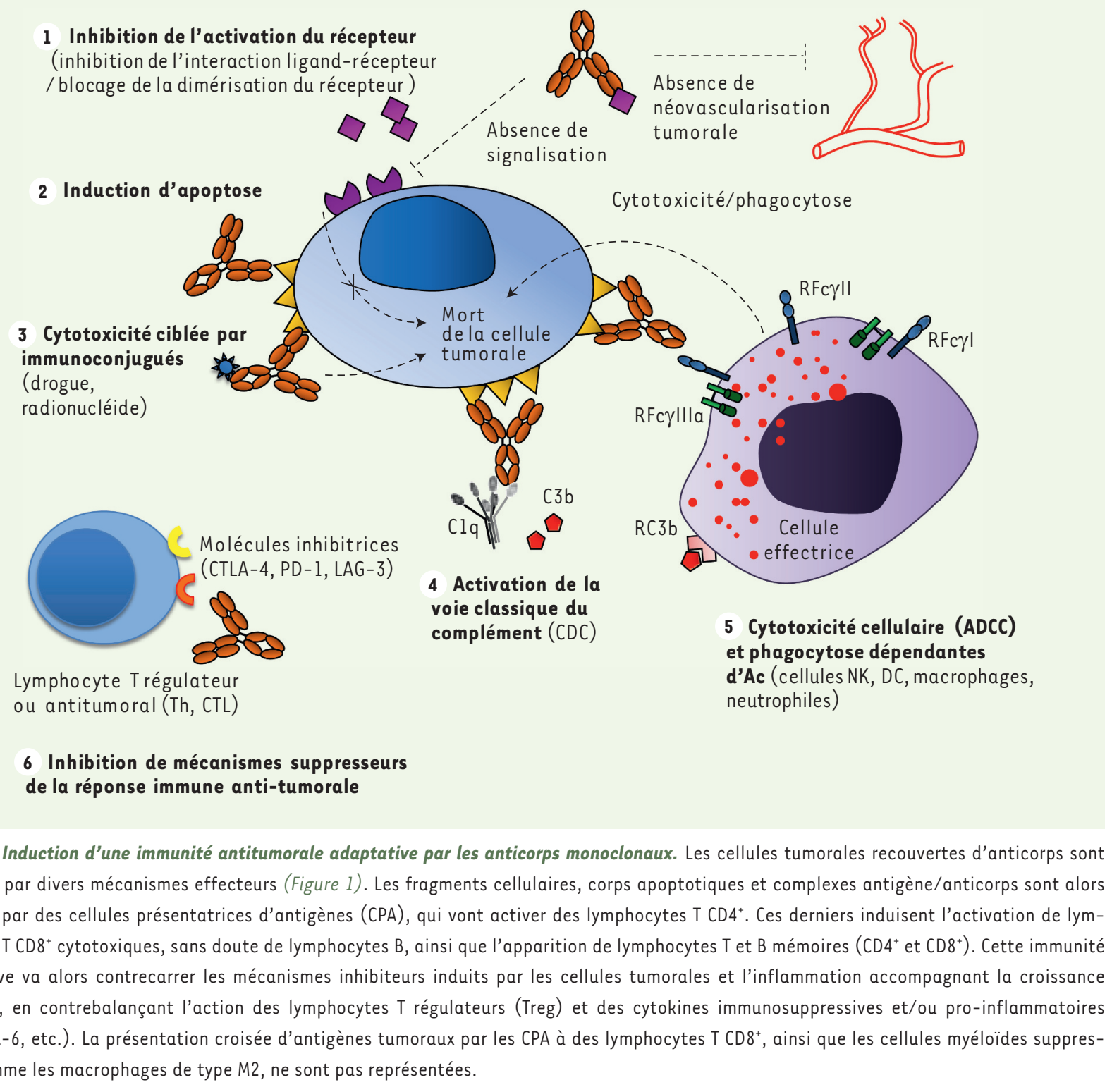

Figure 2. Induction d'une immunité antitumorale adaptative par les anticorps monoclonaux. Les cellules tumorales recouvertes d'anticorps sont détruites par divers mécanismes effecteurs (Figure 1). Les fragments cellulaires, corps apoptotiques et complexes antigène/anticorps sont alors capturés par des cellules présentatrices d'antigènes (CPA), qui vont activer des lymphocytes T CD4 ${ }^{+}$. Ces derniers induisent l'activation de lymphocytes $T C D 8^{+}$cytotoxiques, sans doute de lymphocytes $B$, ainsi que l'apparition de lymphocytes T et B mémoires $\left(\operatorname{CD} 4^{+}\right.$et $\left.\operatorname{CD} 8^{+}\right)$. Cette immunité adaptative va alors contrecarrer les mécanismes inhibiteurs induits par les cellules tumorales et l'inflammation accompagnant la croissance tumorale, en contrebalançant l'action des lymphocytes T régulateurs (Treg) et des cytokines immunosuppressives et/ou pro-inflammatoires (IL-10, IL-6, etc.). La présentation croisée d’antigènes tumoraux par les CPA à des lymphocytes T CD8+, ainsi que les cellules myéloïdes suppressives comme les macrophages de type $M 2$, ne sont pas représentées.

joué par les lymphocytes T CD4 ${ }^{+}$et $\operatorname{CD} 8^{+}$dans le contrôle de la progression tumorale et la survie induite par les Acm.

\section{Le rôle critique des lymphocytes T CD8 ${ }^{+}$}

Ce rôle dans la réponse antitumorale des $A c m$ a été démontré chez des souris porteuses de tumeurs HER2/neu (ErbB-2)+, traitées par une combinaison d'Acm anti-ErbB-2 et anti-death receptor 5 (DR5 ou TRAIL-R2 pour tumor necrosis factor-related apoptosis-inducing ligand-receptor 2), et chez lesquelles les lymphocytes T CD8 ${ }^{+}$avaient été éliminés [4]. Ce rôle des lymphocytes T CD8 ${ }^{+}$dans la réponse clinique aux anti-HER2/neu a été également souligné dans une étude plus récente utilisant des souris $\mathrm{Rag}-\mathrm{I}^{-/-}$ déficientes en lymphocytes $B$ et $T$. Chez ces souris porteuses de tumeurs, un Acm anti-HER2/neu n'a pas permis d'inhiber la progression tumorale [5]. Dans cette même étude, l'élimination des lymphocytes $T \operatorname{CD} 8^{+}$chez des souris immunocompétentes wild type (wt) a conduit au même résultat. La présence des lymphocytes $T$ CD8 ${ }^{+}$est donc indispensable à l'efficacité clinique des anticorps anti-HعR2/neu. Les mêmes auteurs ont alors montré que le traitement par l'Acm anti-HعR2/neu induit des lymphocytes T CD8 ${ }^{+}$spécifiques, producteurs d'interféron- $\gamma($ IFN- $\gamma$ ). Ce mécanisme joue donc un rôle central dans l'induction de la protection antitumorale. Enfin, la réinjection d'un nombre élevé de cellules tumorales chez les animaux traités par l'Acm antiHER2/neu et survivants a révélé l'existence d'une mémoire immunitaire capable de protéger les animaux [5].

\section{Le rôle des lymphocytes T CD4 ${ }^{+}$dans l'induction d'une immunité adaptative antitumorale par des Acm}

La déplétion des lymphocytes $\mathrm{T} \mathrm{CD4} 4^{+}$conduit à l'abolition de la protection antitumorale induite par des Acm 
(anti-gp70 du virus de la leucémie murine de Friend [6] et anti-CD20 [7]) (Figure 2). En 2010, notre équipe a montré que la survie à long terme de souris immunocompétentes porteuses de tumeurs $\mathrm{CD}_{20} \mathrm{O}^{+}$disséminées et traitées avec un $A c m$ anti-CD20 dépendait de la présence de cellules $\mathrm{CD}^{+}[7]$. Des expériences de déplétion ont montré que les CD4 $4^{+}$étaient nécessaires à l'effet antitumoral tant au début du traitement par l'anti-CD20 que lors de la réinjection de cellules tumorales $\mathrm{CD}_{20} \mathrm{O}^{+}$à des souris survivantes à long terme. Ce modèle expérimental a permis également de montrer l'existence d'une mémoire immunitaire : le transfert de cellules $\mathrm{CD}^{+}$provenant d'animaux traités par I'Acm anti-CD20 et isolées une vingtaine de jours après l'injection des cellules tumorales protégeait des animaux porteurs de tumeurs mais non traités par l'Acm anti-CD20. De plus, le transfert de lymphocytes provenant d'animaux traités et survivants après une réinjection de cellules tumorales a permis également d'assurer une protection antitumorale, indiquant l'existence d'une mémoire immunitaire [7]. Enfin, l'injection conjointe d'interleukine-2 (IL-2), si elle n'accroît pas le taux de survie lorsqu'elle est injectée au début du traitement, l'accroît significativement lorsqu'elle est administrée simultanément à la réinjection de cellules tumorales chez les animaux survivants [7]. Un autre travail récent utilise un anticorps trifonctionnel dirigé, d'une part, contre le disialoganglioside $\mathrm{GD}_{2}$ surexprimé notamment par les cellules de mélanome et de neuroblastome, d'autre part, contre la molécule CD3 des lymphocytes $T$, et capable enfin de se fixer par sa région $\mathrm{Fc}$ à des cellules présentatrices d'antigène. Ce travail démontre que ce format d'anticorps permet l'induction de lymphocytes T spécifiques de la tumeur [8].

\section{Les Acm antitumoraux : une biothérapie active}

Toutes ces données suggèrent donc que les anticorps à usage thérapeutique ciblant les cellules tumorales sont capables d'induire une protection à long terme du fait de leur capacité à induire une réponse adaptative spécifique de type mémoire, une véritable vaccination curative en quelque sorte [9] (Figure 2). C'est une rupture complète avec l'idée, encore répandue, selon laquelle l'utilisation thérapeutique des Acm en oncologie représenterait une sorte d'immunothérapie passive à effet immédiat par le seul recrutement de quelques mécanismes effecteurs de l'immunité innée. Cette idée a longtemps exclu le champ des Acm de la catégorie des biothérapies qui est fondée sur l'induction d'une immunité active et adaptative (manipulation ex vivo ou/et in vivo des lymphocytes T, des NKT [natural killer T cells], des cellules dendritiques, etc.).

Cette hypothèse, si elle est confirmée par de nouvelles données expérimentales et cliniques, pourrait avoir d'importantes conséquences sur la façon dont les traitements par Acm doivent s'articuler avec d'autres traitements anticancéreux, chimiothérapie et/ou radiothérapie. Ces derniers sont en effet susceptibles d'inhiber la réponse immunitaire antitumorale du fait de la radiosensibilité des cellules de l'immunité, notamment adaptative, ou de l'effet cytotoxique des drogues. C'est ce qui a pu être démontré lorsque des souris porteuses de tumeurs HER2/ neu $^{+}$ont été traitées, soit avec un anticorps anti-HER2/neu seul, soit en associant cet anticorps à la doxorubicine, soit en associant cet anticorps à un traitement par le cyclophosphamide ou le paclitaxel, administré trois à cinq jours plus tard [5]. Les régressions tumorales les plus fortes ont été observées dans la dernière situation, alors que l'effet antitumoral per se de l'anticorps anti-HER2/neu a diminué lorsque celui-ci était injecté simultanément à la doxorubicine. Cependant, l'utilisation du cyclophosphamide ou du paclitaxel affecte négativement la mémoire immunitaire. Ainsi, lorsque les souris étaient traitées d'abord avec l'anticorps, puis avec l'une de ces deux drogues et recevaient ensuite des cellules tumorales, une progression tumorale était observée ; au contraire, les souris traitées par l'anticorps seul et survivantes résistaient parfaitement à cette réinjection ultérieure de cellules tumorales. À l'inverse, I'utilisation du paclitaxel $24 \mathrm{~h}$ avant le traitement par l'anticorps anti-HER2/neu a conduit à un effet synergique contre la tumeur primaire, et n'a pas affecté la mémoire immunitaire antitumorale, comme l'ont montré des expériences de réinjection de cellules tumorales chez les souris survivantes [5]. Ces travaux montrent donc que le débat sur la recherche d'une fenêtre thérapeutique permettant l'utilisation optimale de drogues antitumorales et d'anticorps antitumeurs doit désormais être ouvert.

\section{Les anticorps monoclonaux en oncologie : inhiber les inhibiteurs}

Cette surveillance des tumeurs par le système immunitaire, qui s'exerce par le biais de cellules de l'immunité adaptative [2], peut être contrecarrée, ou du moins fortement limitée, par des mécanismes suppresseurs. Ceux-ci font intervenir des lymphocytes $T$ régulateurs (Treg), des cellules myéloïdes suppressives (comme les macrophages M2), et/ou l'expression de molécules inhibitrices (cytotoxic T-lymphocyte antigen 4 [CTLA 4, CD152], programmed cell death protein-1 [PD-1, CD279], lymphocyte-activation gene 3 [LAG-3, CD223], etc.) à la surface de lymphocytes T. Différents Acm ont été récemment développés qui inhibent l'action de ces molécules et de ces cellules inhibitrices dans l'espoir de libérer l'immunité antitumorale. La stratégie la plus simple consiste à utiliser des anticorps antagonistes bloquant l'action de molécules inhibitrices (Figure 1). Ainsi ipilimumab, un Acm humain (obtenu en immunisant des souris humanisées) dirigé contre CTLA-4, a reçu une AMM en 2011 dans le mélanome métastatique, où il a permis d'obtenir une amélioration de la survie de plusieurs mois $[10,11]$. Différents essais cliniques sont actuellement en cours avec des anticorps antagonistes dirigés contre d'autres molécules inhibitrices, comme PD-1 [12]. Mais d'autres stratégies sont également 
explorées dans des essais cliniques ou des modèles précliniques: elles visent notamment à combiner des anticorps antagonistes dirigés contre des molécules inhibitrices comme CTLA-4 ou PD-1, soit avec des anticorps agonistes capables de stimuler des molécules activant le système immunitaire (comme CD137, une molécule appartenant à la superfamille des récepteurs du TNF [tumor necrosis factor]) [13], soit avec des anticorps capables de recruter des mécanismes cytotoxiques après avoir lié des molécules exprimées par les cellules tumorales comme CD20 ou $\varepsilon r b B-2$. Il a été montré, par exemple, que I'utilisation conjointe d'un anticorps anti-ErbB-2 et d'un anticorps antagoniste anti-PD-1 ou d'un anticorps agoniste anti-CD137 améliorait l'efficacité thérapeutique de l'anti-عrbB-2 [12]. L'effet bénéfique de cet anti-ErbB-2 requiert l'action de lymphocytes T CD $8^{+}$producteurs d'IFN- $\gamma$, action qui est accrue par l'activation de CD137 ou l'inhibition de PD-1. En utilisant un modèle de xénotransplantation, l'équipe de R. Lévy a de plus montré que le traitement par l'anticorps anti-HER2/neu trastuzumab induit une expression de CD137 à la surface des cellules NK ; un anticorps agoniste anti-CD137 permet alors d'induire une forte activité cytotoxique des cellules NK, même contre des cellules tumorales résistantes à l'action du trastuzumab [14]. La seule utilisation d'anticorps anti-CD137 est d'ailleurs actuellement évaluée chez des patients présentant différentes tumeurs solides (mélanomes, cancers du poumon, cancers de l'ovaire). Il a été montré - dans un modèle de souris immunocompétentes - qu'un anticorps agoniste anti-CD137 permet d'inhiber la croissance d'un lymphome in vivo et d'améliorer la survie des animaux, grâce au développement d'une immunité antitumorale s'inscrivant dans la durée. De plus, cet effet est renforcé par la déplétion des cellules Treg grâce à un anticorps dirigé contre le récepteur

$(\rightarrow)$ Voir $m / s, 2012$, vol. $28, n^{\circ} 5$, page 481 du folate-4 (FR4) [15]. Une autre stratégie actuellement explorée consiste à réévaluer l'effet thérapeutique de cytokines dont le développement a été arrêté du fait d'effets secondaires sévères aux doses utilisées, en les combinant avec des anticorps antagonistes. Ainsi, le traitement par l'IL-15, cytokine dont on sait qu'elle stimule l'activité antitumorale des lymphocytes T CD8 ${ }^{+}$et des cellules NK et NKT, permet de prolonger la survie de souris porteuses de tumeurs de la prostate. Cet effet est observé seulement si l'IL-15 est associée à un anticorps antagoniste bloquant CTLA-4 et à un anticorps bloquant l'interaction du ligand de PD-1 (PD-Ll ou B7-H1, B7 homolog 1, CD274) - exprimé par les cellules tumorales - avec ce récepteur $(\rightarrow)$ [16]. De plus, la fonction suppressive des lymphocytes $T$ régulateurs $C D 4^{+} C D 25^{+}$et $\mathrm{CD} 8^{+} \mathrm{CD} 122^{+}$est diminuée chez les souris qui ont reçu la triple combinaison Acm/IL-15. Ainsi, cette combinaison thérapeutique a permis de rétablir l'activité antitumorale de I'IL-15, et elle ouvre la voie à une utilisation de cette cytokine à des doses plus faibles, permettant d'éviter des effets secondaires indésirables.

\section{Les anticorps monoclonaux en oncologie : le début d'une nouvelle histoire}

Après avoir fait une percée remarquable dans l'arsenal thérapeutique anticancéreux au cours des quinze dernières années (dont témoignait le numéro thématique de médecine/sciences consacré à ce sujet en décembre 2009), les Acm utilisés en oncologie sont désormais perçus non seulement comme exerçant une activité cytotoxique antitumorale immédiate (Figure 1), mais également comme capables d'induire des modifications à long terme au sein des réseaux cellulaires et moléculaires chargés de l'immunosurveillance des tumeurs (Figure 2). L'induction de réponses immunes adaptatives antitumorales permet d'envisager des stratégies thérapeutiques nouvelles, visant à induire une mémoire immunitaire antitumorale, et permettant à l'immunosurveillance des tumeurs de jouer pleinement son rôle. Cette capacité d'induction de réponses adaptatives par des anticorps dirigés contre les tumeurs peut, de plus, être exploitée avec succès en combinant ces anticorps avec des anticorps inhibant les mécanismes suppresseurs de l'immunosurveillance mis en place au cours de la progression tumorale et des réactions inflammatoires qui l'accompagnent. Les essais cliniques en cours reposant sur cette stratégie permettront certainement de mieux évaluer son potentiel. Enfin, cette évolution de notre conception de l'action antitumorale des Acm nous oblige désormais à mieux penser l'articulation entre traitement par Acm, chimiothérapie et/ou radiothérapie, ainsi qu'à réévaluer les stratégies développées jusqu'alors, qui associent traitement par anticorps et immunomodulateurs agissant sur différentes étapes de l'immunité adaptative (facteurs de croissance hématopoïétiques comme le GM-CSF [granulocyte-macrophage colony-stimulating factor], interleukines comme l'IL-2, I'IL-15, etc.). Une nouvelle page de I'histoire des anticorps à usage thérapeutique en oncologie est en train de s'ouvrir. $\diamond$

\section{LIENS D'INTÉRÊT}

Jean-Luc Teillaud déclare avoir participé à des interventions ponctuelles (collaboration pour des travaux scientifiques, conférences) pour l'entreprise LFB.

Claire Deligne déclare n'avoir aucun lien d'intérêt concernant les données publiées dans cet article.

\section{REMERCIEMENTS}

Les auteurs remercient le Dr Riad Abès pour sa contribution au travail de notre équipe auquel il est fait référence dans cet article.

\section{SUMMARY}

The Janus face of monoclonal antibodies in oncology: passive immunity and vaccination

Monoclonal antibodies used in oncology exert direct anti-tumor action leading to cancer cell death. This is due to a variety of mechanisms, ranging from the 
induction of apoptosis to the recruitment of effector cells from the innate immunity. However, antibodies can also induce long-lasting anti-tumor effects thanks to the induction of an adaptive immunity where $C D 4^{+}$and $C D 8^{+} T$ cells play a central role. Different preclinical experimental models, strengthened by a few clinical observations, have shown that, far from being involved only in passive immunotherapy, monoclonal antibodies used in oncology are also endowed with a "vaccine" effect, inducing immune memory, likely responsible for the long-lasting clinical responses that have been sometimes observed. This capacity of triggering/re-installing tumor immune surveillance could be also reinforced by the use, possibly in combination, of antibodies antagonizing molecules such as CTLA-4 or PD-1 that play a key role in the inhibition of the anti-tumor immune responses. Finally, this novel paradigm of therapeutic anti-tumor antibodies as inducers of anti-tumor adaptive immune responses with long-term memory should lead us to re-examine how antibody treatment, chemotherapy, radiotherapy, and biological response modifiers are combined, in particular both in terms of timing and doses. $\diamond$

\section{RÉFÉRENCES}

1. Miller RA, Maloney DG, Warnke R, Levy R. Treatment of B-cell lymphoma with monoclonal antiidiotype antibody. N Engl J Med $1982 ; 306: 517-22$.

2. Fridman WH, Pagès F, Sautès-Fridman C, Galon J. The immune contexture in human tumours: impact on clinical outcome. Nat Rev Cancer 2012 ; 12 : 298-306.

3. Taylor C, Hershman D, Shah N, et al. Augmented HER-2 specific immunity during treatment with trastuzumab and chemotherapy. Clin Cancer Res 2007 ; 13 : 5133-43.

4. Stagg J, Sharkey J, Pommey S, et al. Antibodies targeted to TRAIL receptor-2 and ErbB-2 synergize in vivo and induce an antitumor immune response. Proc Natl Acad Sci USA 2008 ; 105 : 16254-9.

5. Park SG, Jiang Z, Mortenson ED, et al. The therapeutic effect of anti-HER2/neu antibody depends on both innate and adaptive immunity. Cancer Cell $2010 ; 18: 160-70$.

6. Sala A, Gresser I, Chassoux D, et al. Inhibition of Friend leukemia cell visceral metastases by a new monoclonal antibody and role of the immune system of the host in its action. Cancer Res $1992 ; 52: 2880-9$
7. Abès $R$, Gélizé $\varepsilon$, Fridman WH, Teillaud JL. Long-lasting antitumor protection by anti-CD20 antibody through cellular immune response. Blood 2010;116 : 926-34.

8. Eissler N, Ruf $P$, Mysliwietz, et al. Trifunctional bispecific antibodies induce tumor-specific T cells and elicit a vaccination effect. Cancer Res $2012 ; 72$ : 3858-66.

9. Cartron G, Watier H, Golay J, Solal-Celigny P. From the bench to the bedside: ways to improve rituximab efficacy. Blood $2004 ; 104: 2635-42$.

10. Hodi FS, O'Day SJ, McDermott DF, et al. Improved survival with ipilimumab in patients with metastatic melanoma. N Engl J Med 2010 ; $363: 711-23$.

11. Robert C, Mateus C. Anticorps anti-CTLA-4: une avancée thérapeutique majeure dans le traitement du mélanome métastatique. Med Sci (Paris) $2011 ; 27: 850-8$.

12. Berger R, Rotem-Yehudar $R$, Slama G, et al. Phase I safety and pharmacokinetic study of CT-011, a humanized antibody interacting with PD-1, in patients with advanced hematologic malignancies. Clin Cancer Res $2008 ; 14: 3044-51$.

13. Stagg J, Loi S, Divisekera U, et al. Anti-ErbB-2 mAb therapy requires type I and II interferons and synergizes with anti-PD-1 or anti-CD137 mAb therapy. Proc Natl Acad Sci USA 2011 ; 108 : 7142-7.

14. Kohrt $H E$, Houot $R$, Weiskopf $K$, et al. Stimulation of natural killer cells with a CD137-specific antibody enhances trastuzumab efficacy in xenotransplant models of breast cancer. J Clin Invest 2012; 122 : 1066-75.

15. Houot R, Goldstein MJ, Korht HE, et al. Therapeutic effect of CD137 immunomodulation in lymphoma and its enhancement by Treg depletion. Blood 2009; 114 : 3431-8.

16. Yu P, Steel JC, Zhang M, et al. Simultaneous inhibition of two regulatory T-cell subsets enhanced Interleukin-15 efficacy in a prostate tumor model. Proc Natl Acad Sci USA $2012 ; 109$ : 6187-92.

17. Beck A, Wagner-Rousset $\varepsilon$, Wurch T, Corvaia N. Anticorps thérapeutiques et dérivés : une palette de structures pour une pléthore d'indications : quel format et quelle glycosylation choisir? Pour quelles applications. Med Sci (Paris) $2009 ; 25: 1024-32$.

18. Watier H. De la sérothérapie aux anticorps recombinants «nus »: plus d'un siècle de succès en thérapie ciblée. Med Sci (Paris) 2009 ; 25 : 999-1009.

19. Abès $R$, Dutertre CA, Teillaud JL. Les anticorps : mieux les connaître pour mieux s'en servir. Med Sci (Paris) $2009 ; 25: 1011-9$.

\section{TIRÉS À PART}

C. Deligne

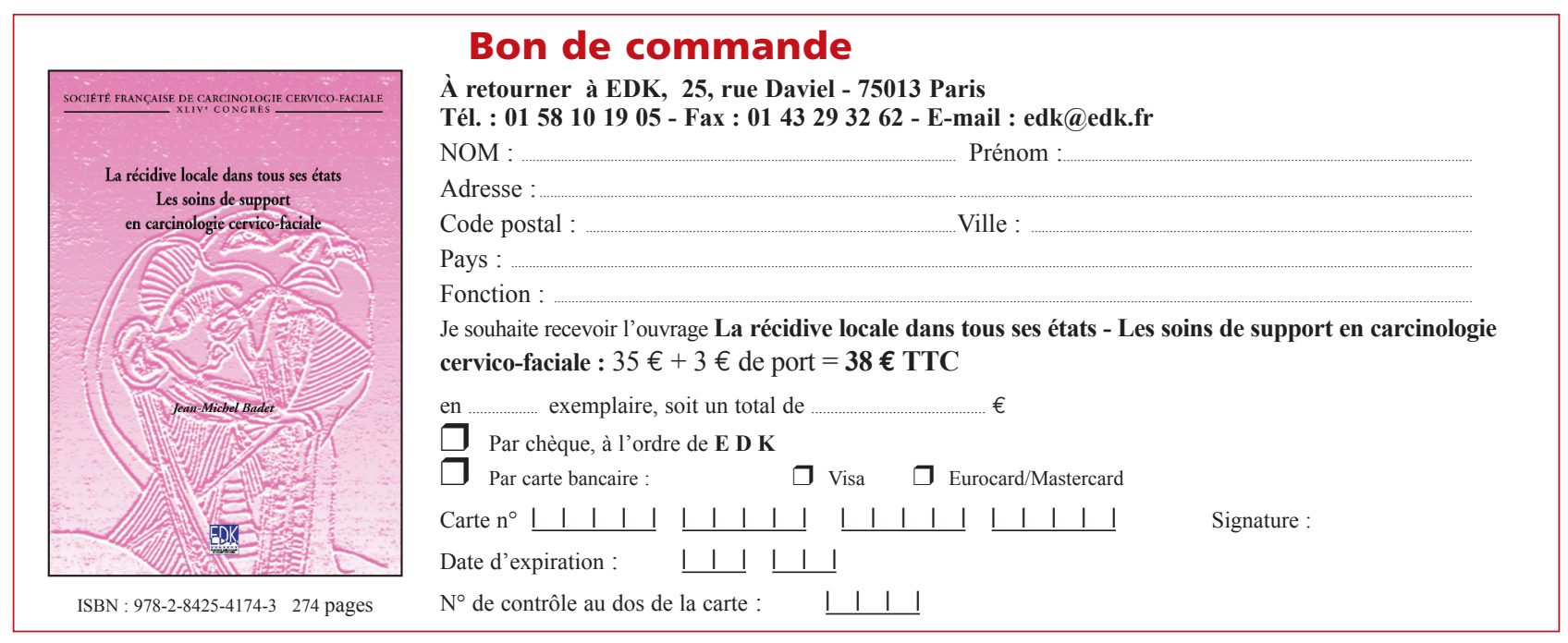

